\title{
Prevision of Head and Neck Cancer Survivorship Care during the 2019 Novel Coronavirus Pandemic
}

\author{
Marci Lee Nilsen, PhD., RN, CHPN ${ }^{1}$ \\ ${ }^{1}$ University of Pittsburgh
}

April 29, 2020

\begin{abstract}
The 2019 Coronavirus Panademic challenges the delivery of care for patients with head and neck cancer. An important aspect of this care has been the evolution of enhanced survivorship services which include surveillance for recurring cancer and prevention of second primaries. The application of evidence based approaches to identification and management of treatment and tumor related toxicities has embraced the use of validated patient reported outcomes instruments (PROs), health promotion, and care coordination. In this manuscript we describe how our multidisciplinary team of survivorship providers have accommodated to the need to provide patients with social distancing while acknowledging the importance of continued care during treatment and through the spectrum of survivorship.

Marci Lee Nilsen, PhD, RN, CHPN, (Mlf981@pitt.edu) Assistant Professor, University of Pittsburgh, School of Nursing; David Clump, MD, PhD (clumpda2@upmc.edu), Assistant Professor, University of Pittsburgh, School of Medicine; Mark Kubik, MD (kubikmw2@upmc.edu), ), Assistant Professor, University of Pittsburgh, School of Medicine; Karen Losego, PT, DPT, CLT-LANA (peltzkj@upmc.edu), University of Pittsburgh Medical Center; Alyssa Mrozek (mrozekac@upmc.edu) University of Pittsburgh Medical Center;Elizabeth Pawlowicz, DMD (pawlowiczec2@upmc.edu), Assistant Professor, University of Pittsburgh, School of Dental Medicine; Debra Pickford, RN (PickfordDM@upmc.edu) University of Pittsburgh Medical Center; Shaum Sridharan, MD (sridharans2@upmc.edu), Assistant Professor, University of Pittsburgh, School of Medicine; Katie Traylor, DO (traylorks@upmc.edu), Assistant Professor, University of Pittsburgh, School of Medicine; Tamara Wasserman-Wincko, MS, CCC-SLP, (wassermantl@upmc.edu) Director of Speech-Language Pathology Division, University of Pittsburgh, School of Medicine; Kelly Young, SLP (youngkh@upmc.edu) University of Pittsburgh Medical Center; Dan Zandberg, MD (zandbergdp@upmc.edu), Associate Professor, University of Pittsburgh, School of Medicine; Jonas T. Johnson, MD (johnsonjt@upmc.edu), Professor, University of Pittsburgh, School of Medicine
\end{abstract}

CORRESPONDING AUTHOR: Marci Lee Nilsen, PhD., RN, CHPN Assistant Professor Department of Acute and Tertiary Care - the University of Pittsburgh, School of Nursing 3500 Victoria Street, 318A Victoria Building, Pittsburgh, PA 15261 412-648-3027 Mlf981@pitt.edu

KEYWORDS: Coronavirus; COVID-19; Cancer; Survivorship; Head and Neck; Treatment Effects

DISCLOSURE STATEMENT: The authors have no conflicts of interest to declare.

ACKNOWLEDGEMENTS: NONE

FUNDING: Beckwith Institute 


\section{Introduction}

In 2006, the Institute of Medicine's (IOM) report, From Cancer Patient to Cancer Survivor: Lost in Transition, called attention to the need for a structured approach to the care of cancer survivors. ${ }^{1}$ The need for surveillance for recurrent cancer, as well as prevention of second primaries, has always been essential. However, the report emphasized that cancer survivors experience a multitude of physical and psychosocial consequences stemming from both cancer and its treatment. The IOM report recommends healthcare providers utilize systematic, evidence-based approaches to the identification and management of these treatments. ${ }^{1}$ Based on a systematic review and consensus expert opinion, the American Cancer Society has provided healthcare providers with clinical practice guidelines for the care of head and neck cancer survivors. These guidelines address five key areas: 1) surveillance for recurrence, 2) screening for second primary cancers, 3) assessment and management of physical and psychosocial effects of cancer and treatment, 4) health promotion, and 5) care coordination. ${ }^{2}$ In addition to endorsing these clinical practice guidelines, the American Society of Clinical Oncology emphasized the importance of team-based, collaborative care to address the complex needs of HNC survivors. ${ }^{3}$ While models of survivorship care delivery vary greatly, the overall goal of these programs is to provide comprehensive and tailored follow-up care. ${ }^{4}$

To optimize the quality of care we provide our HNC survivors, UPMC opened a multidisciplinary, HNCspecific survivorship clinic in December 2016. To tailor care, every patient evaluated in this clinic is surveyed with validated patient-reported outcomes questionnaires (PROs), including the University of Washington Quality of Life. Based on the PROs and patient assessment, the visit is personalized to provide an evaluation of recurrence, prevention of second primaries, identification of treatment-effects, and, subsequently, coordination of care. Our initial report of 238 patients with cancer of the oral cavity, oropharynx, and laryngopharynx demonstrated that over half of patients reported at least three treatment-related effects that impacted their daily life with in the last seven days. ${ }^{5}$ To date, we had over 1,100 HNC survivors in our clinic, and our observations regarding late and long-term treatment effects have remained consistent. Through our work, we have also highlighted how health literacy, financial toxicity, and other treatment-related effects can impact the quality of life in post-treatment patients. ${ }^{6-9}$

The onset of the 2019 novel coronavirus (COVID-19) pandemic has necessitated social distancing and widespread efforts to curb medical care, which could be reasonably postponed or delayed. Cancer care, including diagnosis, treatment, and management of side effects and toxicities, remains essential, and delays are not appropriate. However, the pandemic has challenged us to meet the needs of our patients in alternative methods in order to maintain safety and limit the spread of the virus. We have highlighted measures our team has taken to transform care for patients across the care trajectory during this unprecedented time.

\section{Surveillance}

\section{Physical Examination}

On March 23, 2020, Pennsylvania's governor issues his first stay at home order, which included the county where UPMC's flagship hospital and cancer center are located. Stay at home orders are meant to flatten the pandemic curve, and for many cancer survivors, monitoring via telemedicine is feasible. However, for many patients, physical examination and, possibly, flexible laryngoscopy may be needed. When a biopsy is required, a gown, eye protection, and N95 mask are used. Many of our newly diagnosed HNC patients will need to be discussed in a multidisciplinary conference. To conduct treatment discussions and planning, we have transitioned to a virtual multidisciplinary conference. ${ }^{10}$

When symptom burden is impacting health, we have selectively invited patients to the HNC Survivorship Clinic for face to face evaluation and coordination of care. Symptoms most likely to result in a recommendation for a face to face visit are increasing pain or deterioration in function. Face masks, eye protection, and gloves are routinely employed. We continue to use functional endoscopic evaluation of swallowing (FEES) selectively employing personal protective equipment. We have been able to continue to have patients provided physical therapy at establishments local to them, and we have provided swallowing therapy using a televideo technology. 


\section{Radiology}

Radiology can be a useful adjunct, but we must use it wisely. Moreover, we should not routinely rely on radiographic imaging alone without physical exam because this provides suboptimal care and exposes several other providers, including radiology technicians, transporters. MRI usage also leads to additional safety concerns as most N95 masks are not MRI safe. The ferromagnetic band needs to be removed and the mask taped down to the face to limit gapping at the nose. If the technologist has not passed the N95 fit testing (or has facial hair) and a COVID+ patient is needed to be scanned, another technologist would need to be recruited. Furthermore, PAPR suits are also not MRI safe and cannot be altered to make it non-ferromagnetic. Finally, if a COVID+ patient should be scanned, the process of wiping down/terminal clean takes approximately two hours.

\section{Medical Oncology and Radiation Oncology}

The COVID-19 pandemic has led to changes in medical oncology and radiation oncology care, including surveillance after completion of treatment, in an effort to still provide the care needed, but also reduce risks as much as possible. Each physician evaluates his/her clinical schedule to determine what patients need to be seen in person, what patients can be triaged to telemedicine, and what patients can be re-scheduled for a clinical appointment at a later date. New consults or active patients are being seen in the clinic with continued care as per normal circumstances. Active patients are those who are currently receiving systemic therapy and/or radiation, or undergoing active recovery from therapy, necessitating in-person evaluation and possible supportive care such as intravenous fluids. The outpatient clinical area has developed a screening process for patients. Patients are screened in the lobby of the building, including those receiving daily radiation therapy, for signs/symptoms of infection, the temperature is taken, and all patients are required to wear a mask. If a patient screens positive, a dedicated location for the treatment team to evaluate the patient is available. Currently, there is no visitor policy, with an exception if needed for communication issues, which applies to some of our patients who have undergone a total laryngectomy. The treatment teams engage those family members who are not able to attend the initial evaluation via telephone or video conferencing. Additionally, for example, for those patients receiving traditional chemotherapy alone in the recurrent/metastatic setting, growth factor is being used more regularly in an effort to avoid immunosuppression. Patients in the long term follow-up are being re-scheduled to a later date if possible, with also delay in surveillance imaging. Patients that fall in between these categories are offered a telemedicine visit at the physician's discretion. If a patient cannot do a telemedicine visit or prefers an in-person visit, the patient is able to come into the clinic for their appointment.

Prior to the COVID pandemic, our goal as a head and neck program has been to offer at least one visit with medical oncology and radiation oncology at the main cancer center in order to help develop the plan and offer clinical trials if applicable for all patients. However, in light of the pandemic, those patients that have expressed interest in being treated more closely to home at a Hillman affiliate are being triaged straight to local providers. An additional strategy to decrease exposure has been that radiation oncology completes a telemedicine visit first. Then the in-person visit occurs when the patient is brought for simulation, with medical oncology meeting the patient on that day as well.

\section{Enhanced Care during Chemoradiation}

The population of patients who have the greatest need for immediate care includes patients currently undergoing radiation therapy with or without chemotherapy and immunotherapy. Approximately 30 to $40 \%$ of HNC patients undergoing radiation treatment experience a toxicity-related hospitalization. ${ }^{11,12}$ We had established a new program of enhanced care that had initially been developed to assess symptoms, including pain, swallowing, and weight loss, twice weekly. This program was designed to have symptom assessment conducted via face to face interactions; however, in light of the pandemic, modifications to the protocol have occurred.

In accordance with our Institutional Review Board, we have suspended recruitment but continue to follow patients that have previously been consented. To maintain social distancing, we modified our bi-weekly 
assessments from meeting our enhanced care patients face to face to scheduling phone visits. Alternatively, participants have asked to complete symptom assessments via survey software (i.e., REDcap), which are reviewed by the research staff, and an update is provided to the oncology care team. While participants have provided preference regarding mechanism of symptom assessment delivery, some participants have been difficult to reach via phone or email. Several obstacles have been identified when transitioning this program to telephone or electronic completion, including the time to complete questionnaires, lack of interest, feeling ill, lack of internet, and residency (e.g., nursing home). While the pandemic has altered clinical practice, it has also halted or slowed the progression of research designed to improve quality of life and symptom management in HNC. As clinical scientists, we need to continue to remain abreast of the pandemic progression and changes to research practices dictated by our institutions.

\section{Nutrition}

The COVID-19 pandemic has changed the clinical nutrition field drastically. Interactions between the dietitian and oncology patients that use to occur predominately in person are now mostly occurring over the phone. Patients can still be seen in person during treatment or clinic visits but on a much more limited basis. For the safety of both patients and staff, dietitians have moved into a mostly remote work routine temporarily. A normal nutrition assessment starts with diet interview on appetite, intake, gastrointestinal (GI) symptoms, weight history, and diet history. Depending on the answers to these areas, the dietitian can then determine appropriate interventions to help increase calorie intake, help with weight changes (usually with a goal of weight maintenance during treatment), and help relieve GI distress or treatment side effects. These interventions are then discussed and explained to patients, and handouts are typically given for the patient to take home. Dietitians can also recommend and manage tube feedings.

While diet interview questions can be asked over the phone, nutrition education can be more challenging. It can be difficult to assess the understanding of recommendations provided and the willingness to make changes. Handouts that are usually provided in person and reviewed during education are now mailed/emailed to patients following education sessions. Some HNC patients have a difficult time talking based on tumor location or medical/surgical interventions (i.e., laryngectomy). This can make telephone communication extremely difficult, especially if the patient lives alone. Utilizing email and other family members can be helpful in these situations. Communication with the interdisciplinary team has also shifted to occur more over the phone/email instead of in person. While this communication is usually sufficient, in-person communication with the medical team tends to provide easier interventions and quicker results. Despite the challenges of remote patient care, utilizing phone communication allows for continued nutrition intervention during this time as well as continuity of care. This experience may lead dietitians to explore further other methods of nutrition counseling (i.e., video appointments); however, the value of in-person contact is always the best method for intervention.

\section{Speech-Language Pathology}

The approach for treating patients with dysphagia in the HNC Survivorship Clinic is shifting more to telepractice since the outbreak of COVID-19. Telepractice has been deemed feasible in HNC patients and other adult patient populations, who need continued support by a speech-language pathologist. ${ }^{13},{ }^{14}$ Initially, telepractice was intended to reach patients in remote areas where speech services were not available. Now it is being used to help patients continue with therapy programs in their homes while limiting exposure to COVID-19. It is important to note that each state has its own policies, laws, and professional regulations regarding the provision of teletherapy by speech-language pathologists.

In the HNC Survivorship Clinic, speech-language pathologists are using telepractice with individuals undergoing definitive or adjuvant treatment. This begins after the initial swallowing evaluation has been completed in the clinic and a treatment plan identified. Weekly sessions are held via televideo to promote eating and swallowing exercise programs, with the primary goal to prevent atrophy of the muscles and to help maintain oral diets throughout the course of treatment. We are also using telepractice to engage with individuals who suffer from long-term treatment-related toxicities that impair swallowing function. In 
addition to implementing a swallowing exercise program, televideo visits allow us to complete a modified version of our typical clinical swallowing assessment to monitor diet tolerance, swallowing function, and use of compensatory strategies to reduce aspiration risk. Without our intervention, these patients may be at risk for developing aspiration pneumonia, malnutrition, or further decline with swallowing function. Individualized treatment programs are established based on patient needs, and close collaboration continues with the physical therapy team.

Speech-language pathologists need to continue to monitor, coach and treat individuals who need our help during and after HNC treatment. COVID-19 has changed the way we are doing things, but we may discover that these new methods will bring added value to what we do.

\section{Physical Therapy}

The COVID-19 pandemic marks the first-time physical therapists (PTs) have been able to use telemedicine in Pennsylvania. As of April 2020, PTs are temporarily able to use telemedicine for evaluations as well as return treatment sessions. This new platform for delivering PT services has proven to be beneficial but also challenging as PT evaluation and treatment is, by its nature, largely manual based.

A PT's ability to evaluate a patient with HNC is severely limited with video telemedicine and impossible with audio-only telemedicine. For example, range of motion cannot be objectively measured, manual muscle testing cannot be completed, and pain and tissue restrictions cannot be assessed. These limitations make it challenging to create an optimal patient plan of care. As a result, we continue to encourage patients with severe limitations post-treatment to consider a one-time evaluation in our clinic for proper assessment and to teach crucial manual skills that can be completed at home. This one-time visit will assure better care long term and demonstrate safe, effective basic manual therapy skills; however, we are increasing their community exposure, so this decision cannot be made lightly. If patients are unable or unwilling to attend or do not have severe limitations, telemedicine evaluation is considered with a focus on estimating motion and tissue restrictions to formulate a plan of care.

Treatment sessions via telemedicine are being accomplished with a focus on reinforcement of home manual skills and a robust home exercise program. PTs are demonstrating self-myofascial release, manual lymph drainage, and trigger point release on themselves, and having patients or loved ones mirror the skill on the patient. Therapists must also be diligent in assessing a patient's form with therapeutic exercise to assure patients are exhibiting proper movement patterns. Manual therapy handouts and home exercise programs are being mailed or emailed to the patients following the visit, and the patient is encouraged to follow up with the PT via phone or email in between the mandatory seven-day window between telemedicine visits.

Despite these pitfalls, telemedicine has proven to be a beneficial option of PT care delivery long term: outpatient physical therapists are now able to watch a patient function in their home environment and assess their ability to modify and complete self-management strategies. Via video or phone, PTs can continue to act as coaches to encourage patients to progress their functional mobility with their own means and equipment. Such a treatment model could serve two particular patient populations well: patients with cancer undergoing radiation or chemotherapy who may be too ill or fatigued to attend therapy in person and patients who live geographically far from a skilled PT provider.

\section{Dental Care}

All dental care was suspended by the Pennsylvania Health Department on March $22^{\text {nd }}$, even for emergencies. This directive was amended to allow only emergency care on March $26^{\text {th }}$, contingent on the dental provider wearing personal protective equipment. This has resulted in a lack of access to dental care in HNC patients, including those who must undergo radiation treatment. We have been able to use panorex and relay information about nonviable dentition so they can be removed at the time of surgery.

Dental medicine's inclusion in the multidisciplinary approach to managing morbidity and mortality of surgical and adjuvant therapies for HNC patients has proven to be a major contributor to enhanced outcomes. Dentists provide care and counsel in many critical ways. Primarily, all patients should undergo a pre-surgical 
evaluation of the dentition, including full mouth radiographs, dental and periodontal diagnosis, and prognosis for each tooth. Teeth that cannot be salvaged with conservative restorative or endodontic therapy should be planned for extraction. Radiation therapy to the head and neck can increase the risk for osteoradionecrosis $(\mathrm{ORN})$ of the jaw. Pre-treatment extractions greatly reduce this risk. Additionally, this pre-treatment evaluation offers the surgical, reconstructive, and maxillofacial prosthetics team the opportunity to plan reconstruction together, affecting better outcomes of care.

Additionally, the dental evaluation is critical to assessing the patient's motivation and compliance based upon discussions with the patient and his or her family. Patient education regarding the need for meticulous personal oral hygiene and three-month follow-up dental cleanings and exams must be stressed. This evaluation appointment allows the dentist to give patients proper expectations for treatment burdens as well as offer support and homecare tips that make side effects more manageable.

To start the path to better outcomes, the dentist should perform prophylaxis, periodontal scaling, simple caries control, and fabrication of fluoride trays. To prevent radiation caries, patients should begin daily fluoride treatment with $1 \%$ neutral sodium fluoride gel in prefabricated trays for five minutes each day and be prescribed prescription-strength fluoride toothpaste. The use of fluoride trays usually continues for life, or until a stable neutral oral $\mathrm{pH}$ is achieved.

Since pandemic protocols have caused most non-emergent dental procedures to be delayed indefinitely, this pre-treatment regimen has been almost completely put on hold. The Centers of Disease Control and Prevention and the American Dental Association, as well as almost all state and local health departments, have adopted severe pain and acute infection treatment only mandates. For HNC patients, this means reducing the above-outlined interventions to telehealth counseling and support regarding home care and managing treatment side effects. The use of prescription-strength toothpaste can and should be implemented for these patients.

The area of most concern is the inability, based on the current state and local restrictions, to perform a complete clinical evaluation of each patient. Radiographic examination is still possible and is being used to diagnose acute infection that will be addressed within current guidelines as well as to coordinate possible extraction of non-infected yet non-restorable teeth at the time of tumor resection. The pre-treatment planning between the surgeons resecting and reconstructing with the maxillofacial prosthodontist who will design restorative appliances can occur as best as possible in this manner as well.

Ideally, extractions should be performed three weeks prior to beginning radiation therapy, and extraction of teeth during radiation therapy should be discouraged and delayed until the completion of treatment with resolution of the oral mucositis. A window of one to four months post-radiation where the risk of ORN is low is still available to the dentist and patient. At this time, we are maintaining contact with patients who we believe will need extractions. We hope that pandemic protocols will be lifted within this post-treatment window.

Regarding initial hygiene appointments, fabrication of fluoride gel trays, and rigorous three-month recalls, at this time, we are providing patients with telehealth check-ins. We will resume aggressive hygiene as soon as pandemic protocols are lifted.

\section{Conclusion}

While these changes have occurred in response to the COVID pandemic, importantly, the ultimate decisions on how to care for our head and neck patients have still been made by our collaborative head and neck program and the individual physicians delivering the care. As such, and with a more limited surge of COVID cases to date in Pittsburgh, we feel we have been able to continue to treat our HNC patients during these challenging times adequately.

The COVID-19 pandemic will likely change health care forever. The opportunity to use modern technology to diagnose, counsel, and improve health care for a spectrum of patients in more broadly distributed geographically may, at the end of the day, be the hidden benefit of this catastrophe. 


\section{References}

1. Institute of Medicine and National Research Council, From Cancer Patient to Cancer Survivor: Lost in Transition, ed. M. Hewitt, S. Greenfield, and E. Stovall. 2006, Washington, DC: The National Academies Press. 534 .

2. Cohen, E.E., S.J. LaMonte, N.L. Erb, et al., American Cancer Society Head and Neck Cancer Survivorship Care Guideline. CA Cancer J Clin, 2016. 66(3), 203-39.

3. Nekhlyudov, L., P.A. Ganz, N.K. Arora, and J.H. Rowland, Going beyond being lost in transition: a decade of progress in cancer survivorship. Journal of Clinical Oncology, 2017. 35(18), 1978.

4. Halpern, M.T., M. Viswanathan, T.S. Evans, et al., Models of Cancer Survivorship Care: Overview and Summary of Current Evidence. Journal of Oncology Practice, 2015. 11(1), e19-e27.

5. Nilsen, M.L., L.J. Mady, J. Hodges, T. Wasserman-Wincko, and J.T. Johnson, Burden of treatment: Reported outcomes in a head and neck cancer survivorship clinic.Laryngoscope, 2019. https://doi.org/10.1002/lary.27801

6. Mady, L.J., L. Lyu, M.S. Owoc, et al., Understanding financial toxicity in head and neck cancer survivors. Oral Oncol, 2019. 95, 187-193.

7. Cramer, J.D., J.T. Johnson, and M.L. Nilsen, Pain in Head and Neck Cancer Survivors: Prevalence, Predictors, and Quality-of-Life Impact. Otolaryngol Head Neck Surg, 2018. 159(5), 853-858.

8. Nilsen, M.L., J. Moskovitz, L. Lyu, et al., Health literacy: Impact on quality of life in head and neck cancer survivors. The Laryngoscope, 2019. https://doi.org/10.1002/lary.28360

9. Nilsen, M.L., L. Lyu, M.A. Belsky, et al., Impact of Neck Disability on Health-Related Quality of Life among Head and Neck Cancer Survivors. Otolaryngol Head Neck Surg, 2020. 162(1), 64-72.

10. Dharmarajan, H., J.L. Anderson, S. Kim, et al., Transition to a Virtual Multidisciplinary Tumor Board during the COVID-19 Pandemic: The University of Pittsburgh Experience. Head \& Neck. 2019. https://doi.org/10.1002/hed.26195

11. Hazelden, L.A., M.J. Newman, S. Shuey, J.M. Waldfogel, and V.T. Brown, Evaluation of the head and neck cancer patient population and the incidence of hospitalization at an academic medical center. Journal of Oncology Pharmacy Practice, 2019. 25(2), 333-338.

12. Ling, D.C., P. Kabolizadeh, D.E. Heron, et al., Incidence of hospitalization in patients with head and neck cancer treated with intensity-modulated radiation therapy.Head \& neck, 2015. 37(12), 1750-1755.

13. Collins, A., C.L. Burns, E.C. Ward, et al., Home-based telehealth service for swallowing and nutrition management following head and neck cancer treatment. Journal of Telemedicine and Telecare, 2017. 23(10), 866-872.

14. Weidner, K. and J. Lowman,Telepractice for Adult Speech-Language Pathology Services: A Systematic Review. Perspectives of the ASHA Special Interest Groups, 2020. 5(1), 326-338. 